\title{
Erratum to: ESR1 mutations affect anti-proliferative responses to tamoxifen through enhanced cross-talk with IGF signaling
}

Luca Gelsomino ${ }^{1}$ Guowei Gu$^{2}$ - Yassine Rechoum ${ }^{2}$ - Amanda R. Beyer ${ }^{2}$ • Sasha M. Pejerrey ${ }^{2}$ - Anna Tsimelzon ${ }^{2}$ - Tao Wang ${ }^{2} \cdot$ Kenneth Huffman $^{3}$. Andrew Ludlow ${ }^{3} \cdot$ Sebastiano Andò ${ }^{1}$ Suzanne A. W. Fuqua ${ }^{2,4}$

Published online: 24 April 2017

(C) Springer Science+Business Media New York 2017

\section{Erratum to: Breast Cancer Res Treat (2016)}

\section{7:253-265}

DOI 10.1007/s10549-016-3829-5

In the original publication of the article, the panel for estrogen receptor (ER) in Fig. 2c was incorrectly published. The corrected Fig. 2 is given in this erratum.

The online version of the original article can be found under doi:10.1007/s10549-016-3829-5.

Suzanne A. W. Fuqua

sfuqua@bcm.edu

1 Department of Pharmacy, Health, and Nutritional Sciences, University of Calabria, Arcavacata di Rende, Cosenza, Italy

2 Lester \& Sue Smith Breast Center, Baylor College of Medicine, One Baylor Plaza, Houston, TX 77030, USA

3 Department of Pharmacology, University of Texas Southwestern Medical Center, Dallas, TX, USA

4 Dan L Duncan Cancer Center, Houston, TX, USA 


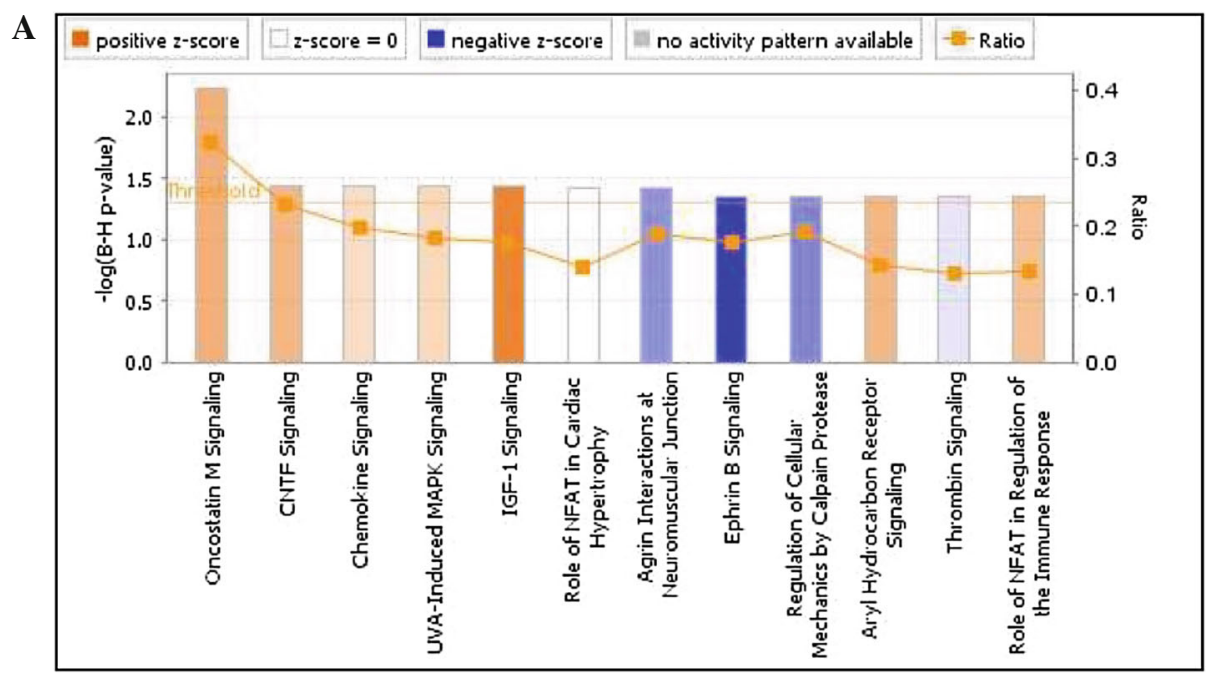

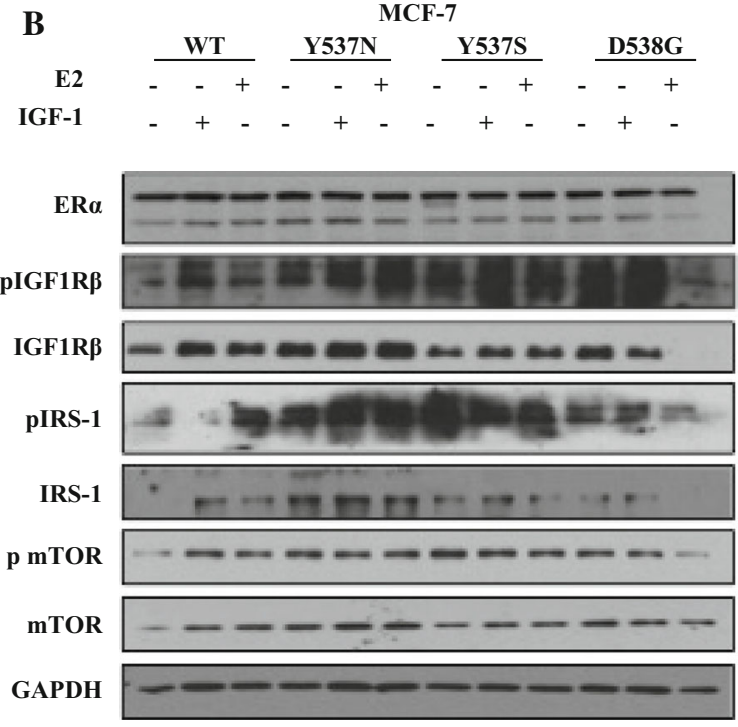

Fig. 2 IGF-1 signaling pathway activation in HBD-ESR1 mutants. a Ingenuity pathway analysis (IPA) to identify activation of signaling pathways in mutant MCF-7 versus ZR-75B. b Total cellular extracts were analyzed for phosphorylation and expression of ER, IGF1R $\beta$, IRS-1, and mTOR; GAPDH was used as a loading control.

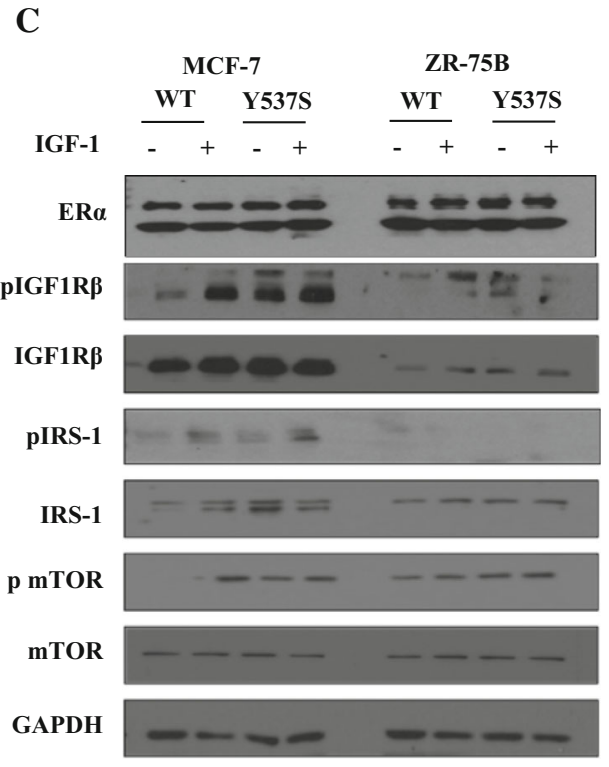

Immunoblots show a representative example of three experiments. c Total cellular extracts were analyzed for phosphorylation and expression of ER $\alpha$, IGF1R $\beta$, IRS-1, and mTOR; GAPDH was used as a loading control. Immunoblots show a representative example of three different experiments 